\title{
Optical properties of polymers and liquid crystals in electrical, magnetic, and hydrodynamic fields
}

\author{
Richard S, Stein ${ }^{1}$, H. Henning Winter ${ }^{2}$, Jöro Müller ${ }^{2}$ and Mohan Srinivasarao ${ }^{3}$ \\ 1 Polymer Research Institute, University of Massachusetts, Amherst, MA, USA \\ 2 Department of Chemical Engineering, University of Massachusetts, Amherst, MA, USA \\ 3 North Carolina State University, Fiber \& Polymer Science Program, Raleigh, NC, USA
}

\begin{abstract}
The behavior of polymer molecules oriented by mechanical, electrical, and magnetic fields is discussed. The consequences of such orientation on optical properties such as birefringence, dichroism, and scattering is analyzed, and means for studying these are illustrated. Particular cases which are described are (a) the change in birefringence dichroism, and light scattering when a crystalline polymer is subject to strain, (b) the change in small-angle neutron scattering when a polymer melt or crosslinked rubber is deformed, (c) the change in birefringence and light scattering accompanying the stress-induced crystallization of a polymer, and (d) the change in light scattering accompanying the shearing of a thermotropic liquid crystal polymer.
\end{abstract}

\section{Introduction}

Polymer molecules are normally anisotropic in their mechanical, electrical, magnetic, and optical properties. As a consequence of their chain structure, their moduli and their electrical and magnetic susceptibilities are tensor quantities leading to anisotropic behavior when the polymer is oriented. Orientation may be brought about through application of an external field which may be rheological, electrical or magnetic. A result will be the development of anisotropic refractive index (birefringence), absorption of radiation (dichroism), fluorescence polarization, scattering and/or diffraction which departs from circular symmetry about the sample normal. Since polymer molecules exhibit longer relaxation times than do small molecules, this optical response will be time dependent, and its study serves to characterize the dynamics of the system.

\section{Polvmer Orientation}

The specification of orientation requires the identification of a particular axis of the molecule which is indicated by a unit vector, $\overline{\mathbf{\theta}}$. This may represent the orientation of the molecule as a whole or that of a particular part of it. For a randomly oriented polymer, all orientations of $\overline{\mathbf{a}}$ are equally probable, whereas particular orientations are favored in an oriented system. The distribution of orientations may be specified by a function $P(\theta, \phi)$ where $\theta$ and $\phi$ are the Eulerian angles of $\overline{\mathbf{a}}$. While it generally depends on both $\theta$ and $\phi$, for uniaxial orientation, it depends only upon $\theta$. Usually, this complete distribution function need not be known, but rather the coefficients of a spherical harmonic expansion suffice (1).

$$
P(\theta, \phi)=P_{0}+P_{2}\left[\left(3<\cos ^{2} \theta>-1\right) / 2\right]+P_{4}\left[\left(5<\cos ^{4} \theta>-1\right) / 4\right]+\ldots
$$

Here, the symbol $<>$ designates an average value. For symmetrical distributions, only the evennumbered harmonics appear. While the series is infinite, the $P_{2}$ and $P_{4}$ terms are usually sufficient for most purposes (2). For example, the aggregate theory of mechanical properties is formulated in terms of these two Pi's. The term, $P_{2}$, has been called the "Hermans orientation function" (3) and sometimes designated by $f$. 
For crystalline polymers, unit vectors, $\overline{\mathbf{a}}, \overline{\mathbf{b}}$, and $\overline{\mathbf{c}}$ may be defined along the three crystal axes, and values of $\mathrm{P}_{\mathrm{i}}$ may be specified for each. For orthorhombic crystals where the axes are perpendicular, it follow that

$$
\left(P_{2}\right)_{a}+\left(P_{2}\right)_{b}+\left(P_{2}\right)_{c}=0
$$

so only two of the three $\left(P_{2}\right)_{i}$ 's are independent $(4)$. Geometric representations of the $\left(P_{2}\right)$ i's have been proposed $(5,6)$.

\section{Polarizability Refractive Index and Birefringence}

The polarizability tensor $|\underline{\alpha}|$ describes the relationship between the induced dipole moment, $\overline{\mathbf{m}}$, and the applied electric field, $\overline{\mathbf{E}}$.

$$
\overline{\mathbf{m}}=|\underline{\alpha}| \overline{\mathbf{E}}
$$

For an isotropic material, $\alpha$ is a scalar so $\overline{\mathbf{m}}$ and $\overline{\mathbf{E}}$ are collinear. In the linear optics approximation, $\overline{\mathbf{m}}$ varies linearly with $\overline{\mathbf{E}}$, but for non-linear optical (NLO) materials, higher powers of $E$ are of concern. The dielectric constant, $\varepsilon$, describes the relationship between the electrical displacement (flux density), $\overline{\mathbf{D}}$ and $\overline{\mathbf{E}}$.

$$
\overline{\mathbf{D}}=|\boldsymbol{\varepsilon}| \overline{\mathbf{E}}
$$

These are related to the polarization, $P$, by

$$
D=E+4 \pi P
$$

where $P=N m=N \alpha E_{i n t}$. In the isotropic case, $P, E, m$, and $\alpha$ are the magnitudes of $\overline{\mathbf{P}}, \bar{E}$, $\overline{\mathbf{m}}$, and $|\alpha|, N$ is the number of molecules $/ \mathrm{cm}^{3}$ and $E_{\text {int }}$ is the internal or local field acting on the molecule. Using the Lorenz approximation of a spherical cavity, this is

$$
E_{\text {int }}=[(\varepsilon+2) / 3] E
$$

By using these relationships and the Maxwell result $\varepsilon=n^{2}$, where $n$ is the refractive index, one obtains the Lorenz-Lorentz equation

$$
\left[\left(n^{2}-1\right) /\left(n^{2}+2\right)\right]=(4 / 3) \pi P
$$

By differentiating, one obtains, for small refractive index difference, the result that the refractive index difference, the birefringence, $\Delta \mathrm{n}$, is related to the polarizability difference, $\Delta P$, by $(7,8)$

$$
\Delta n=\left[(2 / 9) \pi\left(n^{2}+2\right)^{2} / n\right] \Delta P
$$

The "valance bond approximation" assumes that the principal polarizability of a molecule (or other aggregate) may be expressed in terms of the principal polarizabilities of its bonds (or other structural units), $\left(b_{1}\right)_{i}$ and $\left(b_{2}\right)_{i}$ by

$$
P_{A}=\Sigma\left[\left(b_{i}-b_{2}\right)_{i} \cos ^{2} \theta_{11}+\left(b_{2}\right)_{1}\right]
$$

where $\theta_{1 i}$ is the angle between the principal axis of the $i^{\text {th }}$ bond and that of the molecule. This leads to the result for the polarizability difference,

$$
\Delta P=P_{a}-P_{B}=\Sigma\left(b_{i}-b_{2}\right)_{i}\left[\left(3<\cos ^{2} \theta_{1}>-1\right) / 2\right]=\Sigma\left(b_{i}-b_{2}\right)_{i}\left(P_{2}\right)_{i}
$$

so that the birefringence is

$$
\Delta n=\left[(2 / 9) \pi\left(n^{2}+2\right)^{2} / n\right] \Sigma\left(b_{i}-b_{2}\right)_{i}\left(P_{2}\right)_{i}
$$

The relationship between $\left(\mathrm{P}_{2}\right)_{i}$ and the deformation or other field applied to the system depends upon a structural model. For example, the Kuhn-Grün-Treloar model of an ideal rubber consisting of $N_{s}$ identical statistical (Kuhn) segments, each of anisotropy $\left(b_{1}-b_{2}\right)_{s}$, gives for the segment orientation function, $\left(P_{2}\right)_{s}$

$$
\left(P_{2}\right)_{s}=\left[1 /\left(5 N_{U}\right)\right]\left[\lambda^{2}-(1 / \lambda)\right]
$$

where $N_{u}$ is the number of segments per chain (between crosslinks) and $\lambda=L_{L} L_{0}$ is the (uniaxial) extension ratio (where $L$ and $L_{0}$ are the stretched and unstretched lengths of the sample). This gives for the birefringence of a stretched rubber,

$$
\Delta n=\left[(2 / 45) \pi\left(n^{2}+2\right)^{2} / n\right] N_{c}\left(b_{1}-b_{2}\right)_{s}\left[\lambda^{2}-(1 / \lambda)\right]
$$

where $N_{c}=N_{S} / N_{U}$ is the number of chains $/ \mathrm{cm}^{3}$. One might compare this with the kinetic theory of 
rubber elasticity result for the stress, $\sigma$ (based on actual cross-sectional area),

$$
\sigma=N_{c} k T\left[\lambda^{2}-(1 / \lambda)\right]
$$

where $k$ is Boltzmann's constant. The stress-optical coefficient is then

$$
C_{S O C}=\Delta n / \sigma=\left[(2 / 45) \pi\left(n^{2}+2\right)^{2} / n k T\right]\left(b_{1}-b\right)_{) s}
$$

The prediction is that $\mathrm{C}_{\mathrm{SOC}}$ is independent of $\mathrm{N}_{\mathrm{c}}$ (degree of crosslinking), and extension ratio, $\lambda$, and is inversely proportional to the absolute temperature, $T$. These predictions are approxomately in agreement with experiment. The fact that $C_{S O C}$ is independent of $N_{c}$ suggests that it may even have the same value when $N_{c}=0$, as in an uncrosslinked polymer. This is experimentally found to be so and is predicted by current theories of polymer relaxation such as reptation theory. C $\mathrm{C}_{\mathrm{SOC}}$ is a property of the orienting molecule and is independent of the network topology. Indeed, the same value is obtained in a streaming birefringence experiment where the orientation is obtained by hydrodynamically orienting a molecule in solution.

For a multicomponent or multiphase system, one may write (9)

$$
\Delta n=\Sigma(\Delta n)_{i}=\Sigma\left[\Phi_{i}\left(P_{2}\right)_{i}\left(\Delta n_{0}\right)_{i}\right]+\Delta n_{\text {form }}
$$

where $\phi_{i}$ is the volume fraction of component or phase, $\left(P_{2}\right)_{i}$ is its orientation function, and $\left(\Delta n_{0}\right)_{i}$ is its intrinsic birefringence (its birefringence when it is perfectly oriented). $\Delta n_{\text {form }}$ is the "form birefringence" resulting from the distortion of the electrical field by the phase boundary in a multiphase system. The orientations of the various components or phases, $\left(P_{2}\right)_{i}$, are not necessarily the same but depend on the molecular nature and morphology of the system. Thus, the stress-optical coefficient for a binary miscible blend is (10)

$$
\mathrm{C}_{\text {SOC }}=\phi_{1} \kappa_{1}\left(\mathrm{C}_{\mathrm{SOC}}\right)_{1}+\phi_{2} \kappa_{2}\left(\mathrm{C}_{\mathrm{SOC}}\right)_{2}
$$

where the $k_{i}$ 's are weighting factors describing the relative orientation of the two components. For chains of different stiffness, $k$ is larger for the stiffer chain (as can be demonstrated using infrared dichroism as shown in the next section). An approximate theory shows that $k$ is proportional to the square of the Kuhn segment length.

A similar equation holds for a phase-separated blend, except that the $\Delta n_{\text {form }}$, dependent upon the shape of the phase, must be added. In this case, the values of the $\kappa$ 's depend upon the morphology and connectivity of the phase separated regions.

Crystalline polymer obeys this result where (11)

$$
\Delta n=\phi_{c r}\left(P_{2}\right)_{c r}\left(\Delta n_{0}\right)_{c r}+\left(1-\phi_{c r}\right)\left(P_{2}\right)_{a m}\left(\Delta n_{0}\right)_{a m}+\Delta n_{\text {form }}
$$

where $\phi_{c r}$ is the volume fraction crystallinity. This equation may be used to obtain $\left(P_{2}\right)_{a m}$ providing an independent means is available for obtaining $\left(\mathrm{P}_{2}\right)_{\mathrm{cr}}$. This can be done using $\mathrm{x}$-ray diffraction or infrared dichroism. $\Delta \mathrm{n}_{\text {form }}$ may be estimated by swelling the polymer with solvents of differing refractive index, assuming that the solvent enters only the amorphous phase and the orientation is unaffected by swelling (12). When the refractive index of the swollen amorphous phase matches that of the crystal, $\Delta n_{\text {form }}=0$. Such studies indicate that the amorphous contribution to birefringence is less and increases more slowly with elongation than does the crystalline contribution.

An observation is that for stress-induced crystallization (SIC) of polymers, the crystals are usually highly oriented. If one assumes that $\left(P_{2}\right)_{c r}=1$ in such cases, that the SOC for the amorphous phase is that of the completely amorphous polymer, and $\Delta n_{\text {form }}$ is neglected, one obtains

$$
\Delta n=\phi_{c r}\left(\Delta n_{0}\right)_{c r}+\left(1-\phi_{c r}\right) \kappa C_{s o c} \sigma
$$

where $\kappa=\sigma_{\mathrm{am}} / \sigma$ is the fraction of the stress borne by the amorphous phase (often assumed to be 
unity or approximated using composite theory). This gives

$$
\phi_{c r}=\left[\Delta n-\kappa C_{s o c} \sigma\right] /\left[\left(\Delta n_{0}\right)_{c r}-\kappa C_{s o c} \sigma\right]
$$

providing a means for the measurement of $\phi_{\mathrm{cr}}$ from observations of changes in $\Delta \mathrm{n}$ and $\sigma$ during crystallization. This can be done rapidly, and has been reported for the SIC of rubbers and of polyethylene teraphthalate (13).

The dynamic strain-optical coefficient, $K^{\star}$, is defined as $K^{*}=[\partial(\Delta n) / \partial \varepsilon]$ during oscillatory strain, $\varepsilon$. This is a complex quantity which may be resolved into real and imaginary parts (14),

$$
K^{*}=K^{\prime}+i K^{\prime \prime}
$$

where $\delta=\tan ^{-1}\left(K^{\prime \prime} / K^{\prime}\right)$ is phase angle between $\Delta n$ and $\varepsilon$. For a crystalline polymer (15),

$$
K^{*}=\phi_{c r}\left(\Delta n_{0}\right)_{c r}\left[\partial\left(P_{2}\right)_{c r} / \partial \varepsilon\right]+\left(1-\phi_{c r}\right)\left(\Delta n_{0}\right)_{a m}\left[\partial\left(P_{2}\right)_{a m} / \partial \varepsilon\right]+\partial(\Delta n)_{\text {form }} / \partial \theta
$$

Since $\left[\partial\left(P_{2}\right)_{c r} / \partial \varepsilon\right]$ may be measured by dynamic $x$-ray diffraction or dynamic infrared dichroism, the measurement of $K^{*}$ serves to characterize $\left[\partial\left(P_{2}\right)\right.$ am $\left./ \partial \varepsilon\right]$ which provides information about the dynamics of the amorphous phase in crystalline polymers.

This is a particular example of the general dynamic rheo-optical approach and can be applied to the other rheo-optical techniques to be described later. The same formalism can be applied to these as is used for linear viscoelasticity, where a strain-optical spectra function, $B(\ln \tau)$ (where $\tau$ is a relaxation time) may be defined, from which, for example, $K^{\prime}(\ln \omega)$ (where $\omega=2 \pi v$ is the frequency of the experiment) to give (16)

$$
K^{\prime}(\ln \omega)=\int B(\ln \tau)\left[\omega^{2} \tau^{2} /\left(1+\omega^{2} \tau^{2}\right)\right] d \ln \tau
$$

This is analogous to the procedure for determination of the in-phase dynamic modulus $E^{\prime}(\ln \omega)$ from the viscoelasticity relaxation function $H(\ln \tau)(17)$,

$$
E^{\prime}(\ln \omega)=\int H(\ln \tau)\left[\omega^{2} \tau^{2} /\left(1+\omega^{2} \tau^{2}\right)\right] d \ln \tau
$$

For an ideal rubber above its $T_{g}, B(\ln \tau)$ and $H(\ln \tau)$ are proportional, but they are generally not and a comparison indicates how stress relaxation processes relate to orientational relaxation processes.

The birefringence changes become complicated as the glass transition temperature is approached where a "distortional birefringence" contribution occurs which is associated with bond bending and changes in intermolecular distances in the glassy state. Such contributions are not well understood (18).

Orientation can be brought about by electrical and magnetic fields as well as by mechanical and hydrodynamic. Birefringence arising from electrical field orientation is called the Kerr Effect, and its value is proportional to $E^{2}$, with the proportionality constant being the Kerr Constant. It may arise from orienting forces on a molecule arising from both permanent and induced dipole moments (19). Thus, the effect depends upon both the permanent dipole moment and the anisotropy of the polarizability of a molecule. A practical application of the Kerr Effect is for modulating light, where a Kerr cell contains an oriented liquid and is placed between crossed polars. When an electrical field is applied at an angle of $45^{\circ}$ to the polar directions, the resulting birefringence change modulates the light transmission, $T$, since $T=\sin ^{2}(\pi d \Delta n / \lambda)$, where $d$ is the thickness of the cell.

For biaxially oriented samples, the refractive indices along the three principal axes of the sample, $n_{1}, n_{2}$, and $n_{3}$ differ, so three birefringences may be defined: $\Delta n_{1}=n_{2}-n_{3}, \Delta n_{2}=n_{3}-n_{1}$, and $\Delta n_{3}=n_{1}-n_{2}$. Only two of these are independent. For uniaxial orientation, $n_{2}=n_{3}$ and $\Delta n_{1}=0$. For light propagating in the normal, the 3, direction, only $\Delta n_{3}$ is measured. To obtain $\Delta n_{1}$ or $\Delta n_{2}$, either measurements of retardation must be made as a function of the tilt angle, $\phi$, of the sample (20), or else conoscopy may be used in which the sample is observed between crossed polars 
using convergently polarized light. Since the retardation of light rays passing through the sample at different angle will be different, an interference figure results which is characteristic of both $\Delta \mathrm{n}_{1}$ and $\Delta n_{3}(21)$.

Conoscopy may be used for the examination of monodomain liquid crystals which may exhibit homeotropic or planar orientation, leading to characteristic interference patterns. When such samples are sheared, the molecules, and hence their optic axes, tilt, leading to a distortion of the interference pattern permitting establishment of the tilt angle (22).

Liquid crystals are generally stiff molecules having high anisotropic polarizabilities, and thus, high Kerr constants. A practical application of these is for polymer dispersed liquid crystals (PDLC) where low molecular weight liquid crystals are contained within micrometer size cavities in a solid polymer. These generally scatter light because of the difference in refractive indices between the liquid crystal and the polymer matrix. However, with application of an electric field, the liquid crystals become oriented, and, with proper choice, match the refractive index of the matrix, and lead to a decrease in scattering. This can be used for control of light transmission and for displays (23).

In recent studies, PDLCs were observed by conoscopy under magnetic field orientation. The change in interference patterns characterized the change in liquid crystals director orientation distribution within the nematic droplets as well as its time dependence.

\section{Infrared Dichroism}

The absorbance of polarized infrared radiation depends upon the orientation angle, $\theta_{\mathrm{T}}$, of the transition moment, $\bar{M}$, with respect to the polarization direction (24),

$$
A=a \cos ^{2} \theta T
$$

For uniaxial orientation, this leads to the result that

$$
(R-1) /(R+2)=\left[\left(3<\cos ^{2} \theta T>-1\right) / 2\right]=\left(P_{2}\right) M
$$

where $R=A_{\|} / A_{\perp}$ and where $A_{\|}$and $A_{\perp}$ are absorbencies for radiation polarized parallel to and perpendicular to the polymerization direction. Thus, a measurement of $R$ serves to characterize the orientation function of the transition moment, $\left(P_{2}\right)_{M}$. It should be realized that $\left(P_{2}\right)_{M}$ is not necessarily the orientation function of the molecule $P_{2}$ and is related to it by

$$
\left(\mathrm{P}_{2}\right)_{\mathrm{M}}=\mathrm{P}_{2} \bullet\left(\mathrm{PO}_{2}\right)_{\mathrm{M}}
$$

where $\left(\mathrm{PO}_{2}\right)_{\mathrm{M}}$ is the orientation function of the transition moment axis with respect to the molecular axis and has a value requiring knowledge of the transition moment direction.

A significant advantage of the infrared dichroism technique is that it provides a specific measure of $P_{2}$ for the particular part of the structure having an infrared absorption band at the wavelength of measurement. Thus, for example, one can be looking at the orientation of a particular part of a molecule or of a particular component of a copolymer or blend (25). In cases where crystalline and amorphous absorption bands occur at different wavelengths, one can distinguish the orientation of these phases (26). The identification of components can be aided by the isotope labeling technique, since by substituting deuterium for hydrogen in a molecule, infrared bands are shifted to lower frequencies. Thus, selected components or parts of structures can be so labeled (27).

Early examples of the infrared dichroism technique are its use for resolving the orientation functions for the crystalline and amorphous phases of polyethylene (26), polypropylene, and polyethylene teraphthalate (27). It has been used for both miscible and immiscible block copolymers and blends to resolve the orientational response of their components.

The use of Fourier transform infrared spectroscopy (FTIR) permits the rapid acquisition of the entire spectra, so it is helpful for dynamic studies where the observation of changes in several bands are to be simultaneously made. However, for observations at a single wavelength, conventional spectrometers are still preferable. 
In earlier techniques, the measurement of dichroism required separate measurements of $A_{\|}$and $A_{\perp}$ in order to take their ratio so as to obtain $R$. However, now one may employ piezoelectric modulation which can substantially increase the precision of measurement and which renders dynamic infrared dichroism a practical technique (29).

A disadvantage of the infrared dichroism technique is that infrared absorption is strong for many polymers, so it is necessary to employ very thin samples, preventing its use for many samples of practical thicknesses. A way around this problem is to employ the attenuated total reflection (ATR) technique in which the attenuation of surface reflected infrared bands is observed (30). The geometry of such measurements is more complex, and they suffer from the fact that the orientation measured is that of a thin surface layer which may differ from that of the bulk sample.

Another approach which provides complementary information is that of Raman spectroscopy as discussed in a later section.

\section{Polarized Raman Spectroscopy}

When light impinges on a sample, a portion is scattered, mostly at the frequency of the incident light (Rayleigh scattering) but with a small fraction shifted upward or downward in frequency because of the gain or loss of energy of the photons from interaction with quantized molecular energy levels. Thus, such shifts, referred to as "Raman spectra" (31), are measures of these energy levels and relate to the vibrational modes of the molecules. The selection rules for Raman spectra differ from those for infrared (IR) in that for an IR band to be active there must be a dipole moment change accompanying the vibration, whereas for Raman spectra, there must be a polarizability change. Thus, while a molecule like $\mathrm{H}-\mathrm{H}$ is not active for infrared absorption, since there is no dipole moment change accompanying vibration, it is active in Raman scattering since its polarizability does change. It is instructive to consider the infrared and Raman activity of the various vibration modes of $\mathrm{CO}_{2}$. The symmetrical stretching mode,

$<-O=C=O-->$, is inactive in the IR since the dipole moment changes arising from the change in $\mathrm{C}=\mathrm{O}$ bond lengths are equal and opposite, so they cancel. However, the polarizability changes are additive, so the mode is Raman active. On the other hand, for the asymmetric stretching, $\mathrm{O}=\mathrm{C}=\mathrm{O}$, the dipole moment changes for the two $\mathrm{C}=\mathrm{O}$ bonds are additive, whereas the $\rightarrow<-$

polarizability changes cancel, since that for one on the $\mathrm{C}=0$ bonds increases while the other decreases. Thus, this mode is inactive in the IR but active in the Raman. This illustrates the general principle that for molecules such as this having a center of symmetry, the activities in IR and Raman are complementary. Those modes active for one are inactive for the other. It is noted that the transition moment for this mode is parallel to the molecular axis, whereas

$$
\uparrow
$$

that for the bending vibration $\downarrow O=C=O \downarrow$ (active in the IR), is perpendicular to the molecular axis.

Raman scattering involves two successive processes, (a) the absorption of radiation by the molecule dependent upon $(\overline{\mathbf{M}} \cdot \overline{\mathbf{P}})^{2}$, and its emission, dependent upon $(\overline{\mathbf{M}} \cdot \overline{\mathbf{A}})^{2}$, where $\bar{M}$ and $\overline{\mathbf{M}}^{\prime}$ are the transition moment vectors for absorption and emission, respectively, and $\overline{\mathbf{P}}$ and $\overline{\mathbf{A}}$ are unit vectors along the polarization directions of the polarizer and the analyzer, respectively. Thus, the intensity of the Raman scattering is (32):

$$
\text { IRaman }=K_{\text {Raman }}(\bar{M} \cdot \overline{\mathbf{P}})^{2}(\bar{M} \cdot \cdot \overline{\mathbf{A}})^{2}
$$

Usually, the lifetime of the excited state is short compared with characteristic times for molecular motion, so that $\bar{M}^{\prime}=\bar{M}$. For the case where both the polarizer and analyzer are vertically oriented (along the unit vector $\overline{\mathbf{k}}$ lying along the $Z$ axis), the intensity [designated ( $\left.I_{\text {Raman }}\right) \mathrm{Vv}$ ] is

$$
\left(I_{\text {Raman }}\right)_{V v}=K_{\text {Raman }}(\bar{M} \cdot \bar{k})^{4}=K_{\text {Raman }} M^{4} \cos ^{4} \theta_{M}
$$

where $\theta_{M}$ is the angle between $\bar{M}$ and the $Z$ axis. For a distribution of orientations, $\cos ^{4} \theta_{M}$ is replaced by its average value, $\left\langle\cos ^{4} \theta_{M}>\right.$. Thus, this measurement provides information about the fourth moment of the orientation related to the fourth-order spherical harmonic, $\mathrm{P}_{4}$ which cannot be obtained from infrared dichroism. 
When the polarizer and analyzer are crossed, then for scattering at $90^{\circ}$, $\left(\bar{M}^{\prime} \cdot \bar{A}\right)=(1 / 2) M \sin \theta_{M}$, so for this polarization,

$$
\left(I_{\text {Raman }}\right) H v=(1 / 2) K_{\text {Raman }} M^{4} \cos ^{2} \theta_{M} \sin ^{2} \theta_{m}=(1 / 2) K_{\text {Raman }} M^{4} \cos ^{2} \theta_{M}\left(1-\cos ^{2} \theta_{M}\right)
$$

so for a distribution of orientations,

$$
\left(I_{R a m a n}\right) H v=(1 / 2) K_{\operatorname{Raman}} M^{4}\left[\left\langle\cos ^{2} \theta_{M}>-\left\langle\cos ^{4} \theta_{M}>\right]\right.\right.
$$

leading to

$$
\left.<\cos ^{2} \theta_{M}>=\left[1 /\left(K_{\text {Raman }} M^{4}\right)\right]\left[2\left(I_{\text {Raman }}\right) H_{V} \text { - (lRaman }\right) V_{V}\right]
$$

Thus, from measurements with both $V_{v}$ and $H_{v}$ polarization, it is possible to obtain both $P_{2}$ and $\mathrm{P}_{4}$. Such measurements have been extensively employed for the measurement of the orientation of polyethylene teraphthalate (33) where departure from the affine relationship between $P_{2}$ and $\mathrm{P}_{4}$ was seen. With crystalline or phase separated systems, the high Rayleigh scattering can obscure and scramble the polarization of the Raman lines, rendering analysis difficult. However, orientation diminishes the Rayleigh scattering, and satisfactory measurements have been reported for oriented polyethylene samples (34).

As with infrared spectroscopy, the employment of Raman Fourier transform techniques permits simultaneous studies of changes in several Raman bands.

\section{Fluorescence Polarization}

Fluorescence polarization resembles Raman scattering in that it involves the successive absorption and emission of a photon of visible light with the energy exchange with the system. However, in this case, the exchange is with an electronic energy level. Light is usually absorbed in the ultraviolet and emitted in the visible. The geometry is similar, and it is possible to obtain both $\mathrm{P}_{2}$ and $\mathrm{P}_{4}$ from ( $\mathrm{l}_{\text {fluor. }}$ ) $\mathrm{Vv}_{\mathrm{v}}$ and (ifluor.) $\mathrm{Hv}$ measurements (35). Since most polymer molecules are not naturally fluorescent, it becomes necessary to either attach a fluorescent label to the molecule or else add a fluorescent dye. This presents the problem of uncertainty in the relationship between $\bar{M}_{\text {label involved in the measurements and the orientation axis of the }}$ molecule.

Another complication is that the lifetime for the excited state for fluorescence is longer than that for Raman scattering and often comparable with the time scale for molecular motion. Thus, the assumption of the identity of $\bar{M}$ and $\bar{M}$ ' is suspect since orientation may change in between absorption and emission. While this can present a complication in the interpretation of orientation information, it can be used to advantage in learning about molecular motion, and it has been used, for example, to study phase separation of blends (36).

Fluorescence has an advantage in that it utilizes visible light and intensities are usually greater than those for Raman scattering. Thus, fluorescence polarization microscopy is a practical technique.

\section{Scattering - General}

Scattering techniques have the advantage that they depend upon the interference between coherent waves emitted from different parts of the system, so they depend upon its geometry as well as its orientation. In general, the scattered amplitude, $E(\overline{\mathbf{q}})$, is given by (37)

$$
E(\overline{\mathbf{q}})=K_{s} E_{0} \int A(\bar{r}) \exp [i(\overline{\mathbf{q}} \cdot \bar{r})] d^{3} \bar{r}
$$

where $\bar{q}$ is the scattering vector defined by $\bar{q}=(2 \pi / \lambda)\left(\bar{s}_{1}-\bar{s}_{0}\right)$, where $\bar{s}_{1}$ and $\bar{s}_{0}$ are unit vectors along the scattering and incident rays, respectively. The vector, $\overline{\mathbf{r}}$, defines the location of the scattering element, and $d^{3} \bar{r}$ is the volume element for a 3-dimensional integral over all orientations and magnitudes of this vector. The term, exp $[i(\overline{\mathbf{q}} \cdot \overline{\mathbf{r}})]$, represents the phase shift of the incident and scattered wave arising from the element at $\bar{r}$. $A(\bar{r})$ represents the fraction of the incident intensity, $E_{0}$, scattered per unit volume. It depends upon the kind of radiation and the 
properties of the system. If $A(\bar{r})$ is known as a function of $\bar{r}$, then $E(\bar{q})$ may be calculated, and the scattered intensity may be obtained from $I_{s}(\bar{q})=K_{I}\left[E_{s}(\bar{q}) \cdot E_{s}^{*}(\bar{q})\right]$. The inverse of obtaining $A(\overline{\mathbf{r}})$ from $I_{s}(\overline{\mathbf{q}})$ can usually not be uniquely done. Since $A(\bar{r})$ defines the structure of the system, this means that a structure cannot be uniquely determined from scattering, and assumptions or other information must be introduced.

A simplification occurs with spherically symmetrical systems where $A(\overline{\mathbf{r}})$ is independent of the orientation of $\bar{r}$ but depends only upon its magnitude, $r$. In this case, one may integrate over the angular coordinates of $\overline{\mathbf{r}}$, leading to (38)

$$
E_{s}(q)=4 \pi K_{s} E_{0} \int A(r)[\sin (q r) /(q r)] r^{2} d r
$$

The amplitude, $E_{s}(q)$ then depends only upon $q$, the magnitude of $\overline{\mathbf{q}}$, given by $=(4 \pi / \lambda) \sin (\theta / 2)$, where $\theta$ is the "scattering angle" between $\bar{s}_{1}$ and $\bar{s}_{0}$. Thus, the scattering pattern is then circularly symmetrical about $\bar{s}_{0}$. In the simple case of a uniform sphere in $a$. vacuum with radius, $R_{s}$, and constant scattering power, $A_{s}$, this gives,

$$
E_{s}(q)=E_{o} K_{s} A_{s} V_{s} \Phi_{s}(U)
$$

where $V_{S}$ is the volume of the sphere [ $V_{S}=(4 / 3) \pi R_{S}{ }^{3}$ ], and $\Phi_{S}(U)$ is the sphere scattering function defined as

$$
\Phi_{s}(U)=\left(3 / U^{3}\right)(\sin U-U \cos U)
$$

where $U=q R_{S}$. This may readily be generalized to a sphere with a radial variation in $A_{S}(r)$. For a uniform sphere immersed in a medium with scattering power $A_{0}$, this leads for the intensity of scattering to

$$
I_{s}(q)=I_{0} K_{s}^{2} V_{s}^{2}\left(A_{s}-A_{0}\right)^{2} \Phi_{s}(U)^{2}
$$

This predicts that the scattered intensity varies with $V_{s}{ }^{2}$ or $R_{s}{ }^{6}$ so that large spheres scatter much more than small ones. The term, $\left(A_{S}-A_{0}\right)^{2}$, is called the "contrast" and represents the difference between the scattering of the sphere and that of its surroundings. It can be changed by adjusting the nature of the system or its surroundings as can be done in light scattering by changing the refractive index of the surroundings or in neutron scattering by deuterium substitution in one of the components. The scattering from a component may be eliminated by "contrast matching" where one adjusts its $A_{S}$ or $A_{0}$ so as to make $\left(A_{S}-A_{0}\right)=0$. The function $\Phi_{S}(U)$ is an angularly dependent term reffecting the size of the sphere, $R_{\mathbf{S}}$. It oscillates in value representing various orders of interference and decreases more rapidly with increasing $\theta$ for larger $R_{\mathbf{S}}$. Thus, its observation serves to characterize the sphere size. One may sum the intensities from a random collection of spheres to describe the effects of a distribution of sizes.

One may carry out similar calculations for other spherically symmetrical systems and may general show that $(38)$

$$
I_{s}(q)=I_{s}(0)\left[1-\left(R_{q}^{2 / 3}\right) q^{2}+\ldots\right]
$$

where $I_{s}(0)$ is the value of $I_{s}(q)$ at $q=0$. $R_{g}{ }^{2}$ is the radius of gyration of a arbitrary spherically shaped object. Thus a plot of the interference function, $P(\theta)=I_{s}(q) / I_{s}(0)$ vs $q^{2}$ should lead at small $q$ to a linear variation with slope $-R_{q}{ }^{2} / 3$. This approach is conventionally used to determine the $R_{\mathrm{g}}$ of a polymer molecule in dilute solution. It is necessary that the wavelength and $\theta$ range of the measurements be selected so that one can neglect terms of higher powers in $q$ in the above equation.

It is necessary that such measurements be made in dilute solution to avoid contribution from interparticle interference. However, as discussed later, concentrated solution or even bulk measurements of $R_{g}$ are possible using neutron scattering by contrast matching the surroundings by deuterium substitution.

Where the particulate nature of the scattering system cannot be identified, one may describe the scattering in statistical terms using the approach of Debye and Bueche (DB). This recognizes that 
scattering is completely canceled from large homogeneous systems and the scattering from real system arises from fluctuations from homogeneity. They give (39)

$$
I_{s}(\bar{q})=K_{s} V<\eta^{2}>\int \gamma\left(\overline{\mathbf{r}}_{i j}\right) \exp \left[-i\left(\overline{\mathbf{q}} \cdot \bar{r}_{i j}\right] d^{3} \bar{r}_{i j}\right)
$$

where $V$ is the volume of the scattering system and $\eta(\overline{\mathbf{r}})$ is the fluctuation defined as $\eta(\overline{\mathbf{r}})=A(\overline{\mathbf{r}})-\langle A(\overline{\mathbf{r}})\rangle$. $\quad\left\langle\eta^{2}\right\rangle$ is its mean-squared value, and $\gamma\left(\overline{\mathbf{r}}_{i j}\right)$ is a correlation function defined by

$$
\gamma\left(\overline{\mathbf{r}}_{\mathrm{ij}}\right)=\left\langle\eta\left(\overline{\mathbf{r}}_{\mathrm{i}}\right) \eta\left(\overline{\mathbf{r}}_{\mathrm{j}}\right)\right\rangle_{\mathbf{r}_{\mathrm{ij}}} /\left\langle\eta^{2}\right\rangle
$$

The average in the numerator is obtained for all pairs of volume elements, $i$ and $j$, separated by vector distance, $\overline{\mathbf{r}}_{\mathrm{j} j}=\overline{\mathbf{r}}_{\mathrm{i}}-\overline{\mathbf{r}}_{\mathrm{j}}$. The spherically symmetrical version of the DB equation is

$$
I_{s}(q)=4 \pi K_{s} V<\eta^{2}>\int \gamma(r)[\sin (q r) /(q r)] r^{2} d r
$$

for a two-phase system of volume fractions $\phi_{1}$ and $\phi_{2}$ and scattering powers $A_{1}$ and $A_{2}$ having sharp boundaries, $\left\langle\eta^{2}\right\rangle=\phi_{1} \phi_{2}\left(A_{1}-A_{2}\right)^{2}$. The correlation function declines from unity when the separation of the volume elements, $r=0$, toward zero for infinite separation for systems having no long range order in a manner characterizing the geometry of the system. It may be obtained by Fourier inversion of $I_{s}(q)$. For many systems, this has the functional form, $\gamma(r)=\exp \left(-r / a_{c}\right)$ where $a_{c}$ is a correlation distance representing the size of the fluctuating region. It has been shown that this form is rigorous for a random two-phase system having sharp boundaries (40). This function leads to the result that

$$
I_{S}(q)=K_{D B}\left\langle\eta^{2}>a_{c}{ }^{3} /\left[1+q^{2} a_{c}^{2}\right]^{2}\right.
$$

The prediction is that for small $a_{c}$, the scattered intensity increases with $a_{c}{ }^{3}$, but at large $a_{c}$, decreases as $\left(1 / a_{c}\right)$. It passes through a maximum when $a_{c}=1 / q$ expressing the usual conclusion that systems scatter most when their sizes are of the order of the wavelength of the radiation. At large $q$, it predicts that the intensity decreases with $1 / q^{4}$ in agreement with "Porod's Law", a general result for systems having sharp boundaries. $a_{c}{ }^{2}$ may be determined from the slope of a plot of $I_{s}(q)^{-1 / 2}$ vs $q^{2}$, a "DB plot".

The $D B$ theory has been used for characterizing a variety of systems using differing radiation. These include (a) porous rock, (b) catalysts, (c) phase separated blends, and (d) swollen inhomogeneously crosslinked rubbers. It provides a basis for formulating the scattering from liquids and solutions.

The scattering power, $A(r)$, depends on the density and composition of a system. Fluctuations in density depend upon compressibility and go through a maximum at critical points, giving rise to critical opalescence. In solution, additional fluctuations arise from concentration fluctuations to a degree dependent upon the osmotic compressibility, leading to

$$
[l(q)]_{\text {reduced }}=\left[l(q)_{\text {solution }}-[I(q)]_{\text {solvent }} \sim c /(\partial \Pi / \partial c)\right.
$$

where $\mathrm{c}$ is the concentration and $\Pi$ the osmotic pressure. Upon using a virial expansion for $\Pi$, this gives the "Zimm equation" (41),

$$
H C / I_{s}(q)=1 /\left[M_{2} P(\theta)\right]+2 A_{2} c
$$

where $H$ is a constant proportional to $K_{s}, M_{2}$ is the molecular weight of the solute, and $A_{2}$ is the second virial coefficient related to the Flory interaction parameter, $\chi$. This equation, restricted to dilute solution has been extensively used for the measurement of molecular weight and interaction parameters for polymers in dilute solution.

By using the Flory-Huggins theory, or its generalization using the "random phase approximation", one can obtain a more general equation valid at high concentrations of binary solutions as (42)

$$
k^{\prime \prime} / I_{s}(q)=\phi_{1} /\left[z_{1} P i(q)\right]+\phi_{2} /\left[z_{2} P_{2}(q)\right] \cdot 2 \chi
$$

where the $\phi_{i}$ 's and the $z_{i}$ 's are the volume fractions and degrees of polymerization of the two components. While, in principle, this equation permits the determination of $\chi$ from scattering from concentrated solution, it has not been used for light scattering because of the difficulty of cleaning such solutions, but, as will be discussed, it is used for neutron scattering. 
Light Scattering

In the special case of light scattering, $K_{s}$ depends on polarizability, related to the refractive index. In addition to the conventional measurements on dilute solutions, studies have been extended to phase separated systems such as polymer blends. A special feature of light scattering is its dependence upon polarization of the light, arising because of the anisotropy of molecular polarizability. The scattering power is given by (43)

$$
A(\overline{\mathbf{r}})=[\bar{M}(\overline{\mathbf{r}}) \cdot \overline{\mathbf{0}}]
$$

where $\bar{M}(\bar{r})$ is the induced dipole moment at $\bar{r}$ given by

$$
\overline{\mathbf{M}}(\overline{\mathbf{r}})=\delta(\overline{\mathbf{r}})\left[\bar{E}_{0} \cdot \overline{\mathbf{a}}(\overline{\mathbf{r}})\right] \overline{\mathbf{a}}(\overline{\mathbf{r}})+\alpha_{2}(\overline{\mathbf{r}}) \overline{\mathbf{E}}_{0}
$$

$\overline{\mathbf{0}}$ is a unit vector in the direction of the analyzer polar, $\delta(\overline{\mathbf{r}})$ is the anisotropy of polarizability at $\overline{\mathbf{r}}$ (assumed uniaxial) given by $\delta(\overline{\mathbf{r}})=\alpha_{1}(\overline{\mathbf{r}}) \cdot \alpha_{2}(\overline{\mathbf{r}})$ for the principal polarizability directions and $\overline{\mathbf{a}}(\overline{\mathbf{r}})$ is a unit vector in the direction of the principal polarizability direction. This has been used to generalize the equation for the scattering from a sphere, taken as an idealized model for a polymer spherulite. It gives, for example, the prediction of scattering with crossed polarizers $\left(H_{v}\right)$ (44)

$$
\left[I_{s}(q)\right]_{H v}=(1 / 15) K_{s}<\delta^{2}>V_{s}^{2} \Phi_{3}(U) \sin ^{2} \mu \cos ^{2} \mu
$$

where $\mu$ is the azimuthal angle of scattering, and $\Phi_{3}(U)$ is a scattering function defined by

$$
\Phi_{3}(U)=\left(3 / \mathrm{U}^{3}\right)[4 \sin U-U \cos U-3 \mathrm{Si} U \text { ] }
$$

where Si $\mathrm{U}$ is the sine integral. This component of scattering, only seen for anisotropic spheres, exhibits a four-leaf clover appearance with maxima at values of $\mu$ that are odd multiples of $45^{\circ}$ and at $\theta$ given by $(4 \pi / \lambda) \sin \left(\theta_{\max } / 2\right)=4.08$. Such scattering is in good agreement with experiment and provides a means for readily measuring the size of spherulites. Such may be rapidly followed during crystallization (45).

An equation may also be obtained for scattering with parallel polarizers. This has two components, one which is independent of $\mu$ and depends upon the difference between the average polarizability of the spherulite and that of its surroundings, and the other dependent upon the anisotropy of polarizability of the spherulite and varying with $\cos ^{4} \mu$, and thus having two-fold symmetry in $\mu$. For isotropic spheres, this second component is zero and the first component reduces to the equation for scattering from isotropic spheres. At the beginning of crystallization, the spherulite surroundings is amorphous polymer, so the first contribution dominates and the scattering is $\mu$ independent. As crystallization proceeds, the surroundings become other spherulites more closely matching the spherulite in average polarizability, so the first term decreases. However, because of the growth of oriented crystals within the spherulites, their anisotropy increases, so the second term grows leading to the development of two-fold symmetry in the $V_{v}$ pattern.

These measurements may be made in real time during the rapid crystallization of polymers, and thus provide a means for resolving the process into its basic contributions of nucleation, growth, and secondary crystallization. The theory has been extended to describe deformed spherulites and has been used to follow their response to sample stretching (46).

Liquid crystals also exhibit anisotropy of polarizability with correlations extending over distances comparable with the wavelength of light. This leads to scattering in a manner related to the extent of this correlation and to their orientation. Theory has been developed and experiments have been conducted for describing such scattering in terms of the number and arrangement of disclinations and other orientational defects (47). Changes in scattering accompanying orientation by flow, electrical, and magnetic fields have been analyzed. Such changes are basic to the operation of display devices based upon scattering such as polymer dispersed liquid crystals. 


\section{Neutron Scattering}

Neutron scattering follows the same principles that have been discussed, but differs is several aspects: (a) the wavelengths of neutrons are in the range of $0.1-2.5 \mathrm{~nm}$ and permit studies of smaller morphologies than accessible by light scattering and exceed slightly those for $x$-ray scattering, (b) the contrast for neutrons is a nuclear property and can be greatly changed by isotopic substitution (deuterium for hydrogen), (c) neutrons penetrate many substances so apparatus windows can be made of materials such as aluminum, and (d) the rigorous cleaning of systems usually required for light scattering measurements is usually not necessary since the dimensions of impurities are usually greater than those of morphologies being studied so the scatter at smaller angles and their contrast is usually less than that offered by deuterium-labeled molecules (48). Thus, filtering or centrifugation, usually not possible in concentrated solutions or bulk, is not required. These advantages are somewhat offset by the low fluxes of available sources and the difficulties in collimation and of detection.

Deuterium substitution makes possible studies in concentrated solutions and bulk polymers not possible with $x$-ray or light scattering (49). A component may be dilute in deuterium concentration while concentrated thermodynamically. Thus, measurements of molecule radii of gyration and orientation and interaction parameters, $\chi$, under such conditions have been made and studies of the miscibility and phase separation of polymer melts have been conducted (50). The possibility of labeling parts of molecules has permitted studies of block copolymers (51), branched polymers. and bimodal networks (52), where selected components are labeled.

\section{Conclusion}

Rheo-optical techniques may be of great value in polymer science and permit the determination of information not readily accessible by other means. They are an important complement to rheological studies of polymeric systems.

\section{Acknowledament}

The studies reported here were aided by many students, associates and coworkers too numerous to mention. Support came from the National Science Foundation, the Materials Research Laboratory and the Center for University of Massachusetts-Industry Research in Polymers and industry sponsorship by the Exxon, Monsanto and Novacor Chemical Companies..

\section{REFERENCES}

1. F. H. Müller, Koll. Zeit.., 95, 172,307 (1944)

2. I. M. Ward, Mechanical Properties of Polymers, John Wiley \& Sons, New York, 2nd Ed.. 1974

3. P.H. Hermans, Contributions to the Physics of Cellulose Fivers, Elsevier, New York, 1946, p. 198

4. R.S. Stein, $j$. Polymer Sci. , 31, 327 (1958)

5. R.S. Stein, j. Polymer Sci. , 34, 70 (1959)

6. R. S. Stein and C. R. Desper, J. Appl. Phys., 11,3990 (1966)

7. W. Kuhn and F. Grün, Koll. Zeit. , 101, 248 1942)

8. L.R.G. Treloar, The Physics of Rubber Elasticity,, Oxford, Clarendon Press. 1949

9. G. R. Taylor and S. R. Darin, J. Appl. Phys. , 26, 1075 (1955)

10. B. H. Arendt, R. M. Kannan, M. Zewail, J. A. Kornfield and S. D. Smith, Rheol. Acta., 33, 322-336 (1994)

11. R.S. Stein and F. H. Norris, J. Polym. Sci. , 21, 381 (1956)

12. F. A. Bettelheim and R. S. Stein, J. Polym. Sci., 27, 567 (1958)

13. R. S. Stein, M. Hashjiyama and M. K. Parpart, Midland Macromolecular Monographs, Gordon and Reach, Flow-Induced Crystallization In Polymer Systems, ed. by R. L. Miller,6, 331-349 (1979)

14. R. S. Stein, S. Onogi and D. A. Keedy, J. Polymer Sci., 57, 801 (1962)

15. R. S. Stein, R. J. Cembrola, T. Kyu, S. Suehiro and H. Kawai, J. Polymer Sci., Polymer Physics Ed., 20, 1279 (1982)

16. R. S. Stein and R. Yamada, J. Appl. Phys.,10, 3005 (1965) 
17. J. D. Ferry, Viscoelastic Properties of Polymers, John Wiley \& Sons, New York, 1969

18. R. S. Stein, S. Krimm, and A. V. Tobolsky, Textile Res. J., 19, 8 (1949)

19. E. Riande and E. Saiz, Dipole Moments and Birefringence of Polymers, Prentice Hall, NJ (1992)Chap.7

20. R. S. Stein, J. Polym. Sci.., 24, 383 (1957)

21.M. Born and E. Wolff, Principles of Optics, Pergamon Press, New York, 1988, pp. $691-710$

22. P. Pieranski and E. Guyon, Phys. Rev. Lett.., 32, 924 (1974)

23. R. S. Stein \& M. Srinivasarao, Liquid Crystal Materials, Devices, and Applications II, Proc. Int. Soc. Opt. Eng.,1911, 132-151 (1993)

24. Elliot, Ambrose, and Temple, J, Chem, Phys., 16, 877 (1948)

25. S. L. Hsu, F. J. Lu, D. J. Burchell and X. Li , Polym. Eng. and Sci. , 23, 861 (1983)

26. B. E. Read and R. S. Stein, Macromolecules, 1, 116 (1968)

27 A. Hanyu and R. S. Stein, Makromol. Symp. 45, 189 (1991)

28. R.S. Samuels, Structured Polymer Properties, New York, Wiley, 1974

29. I. Noda, CHEMTRACTS, Macromolecular Chemistry Ed., 1, 89 (1990)

30. N. J. Harrick "Internal Reflection Spectroscopy", Harrick Scientific Corpn New York (1979).

31.S. W. Cornell And J. S. Koenig, J. Appl. Phys. 39, 4883 (1968)

32. R. G. Snyder, J. Molec. Spectr, . 37, 353 (1971)

33. D. I. Bower, J. Polym. Sci., Polym. Phys. Ed. , 10, 2135 (1972)

34. R. S. Stein, J. Maxfield and M. C. Chen, J. Polymer Sci., Polymer Physics Ed., 16, 37 (1978)

35. Y. Nishijima, Y. Onogi, T. Tsai, J. Polym. Sci. ,C, 15, 237 (1966)

36. D. Phillips and I. Soutar in Photophysical and Photochemical Tools in Polymer Science., M. Winnick, Ed., NATO ASI Series: Series C, Vol.182

37. R. S. Stein and J. S. Higgins,. J. Appl. Cryst., 11, 346 (1978)

38. A. Guinier, X-Ray Diffraction in Crystals, Imperfect Crystals, and Amorphous Bodies, W. H. Freeman and Co. San Francisco, 1963

39. P. Debye and A. M. Bueche, J. Appl. Phys. 20, 518 (1949)

40. P. Debye, H. Anderson, and H. Brumberger, J. Appl. Phys.. 28, 679 (1957)

41. B. H. Zimm, R. S. Stein, and P. Doty, Polumer Bull., 1, 90 (1945

42. P.G. DeGennes, Scaling Methods in Polymer Physics,, Cornell University Press, Ithaca, 1979

43. R. S. Stein and P. R. Wilson, J. Appl. Phys., 6, 1914 (1962)

44. M.B. Rhodes and R.S. Stein, J. Appl. Phys., 31, 1873 ((1960)

45. R. S. Stein, S. N. Garg, T. K. Su, R. J. Tabar and A. Misra, Kinetics Of Aggregationa And Gelation, ed. by F. Family and D. P. Landau, Norh-Holland, Amsterdam, 229-234, 1984

46. R. S. Stein, S. Clough, and J. J. van Aartsen, J. Appl. Phys., 10, 3072 (1965)

47. R. S. Stein, M. B. Rhodes and R. S. Porter, J. Colloid and Interface Sci., 3, 336 (1968)

48. R. S. Stein and C. C. Han, Physics Today, 38, 74, (1985)

49. R. S. Stein, G. Hadziioannou, L. H. Wang and R. S. Porter, Macromolecules, 15, 880, (1982)

50. F. S. Bates and G. H. Fredrickson, Annu. Rev. Phys. Chem, Vol. 41, 525 (1990)

51. Wen-li Wu, L. D. Coyne, L. Jong, A. Hanyu, and R. S. Stein, Macromolecules, 23, 351-3 (1990) 